\title{
Interplay between Presynaptic and Postsynaptic Activities Is Required for Dendritic Plasticity and Synaptogenesis in the Supraoptic Nucleus
}

\author{
Vivien Chevaleyre, Françoise C. Moos, and Michel G. Desarménien \\ Centre National de la Recherche Scientifique Unité Mixte de Recherche 5101, Biologie des Neurones Endocrines, 34094 \\ Montpellier Cedex 5, France
}

Developing oxytocin and vasopressin (OT/AVP) supraoptic nucleus (SON) neurons positively autocontrol their electrical activity via dendritic release of their respective peptide. The effects of this autocontrol are maximum during the second postnatal week (PW2), when the dendritic arbor transiently increases and glutamatergic postsynaptic potentials appear. Here, we studied the role and interaction of dendritic OT/AVP release and glutamate release in dendritic plasticity and synaptogenesis in SON. In vivo treatment with the peptides antagonists or with an NMDA antagonist suppressed the transient increase in dendritic arbor of SON neurons at the beginning of PW2. Incubation of acute slices with these compounds decreased the dendritic arbor on a short time scale (3-8 hr) in slices of postnatal day 7 (P7) to P9 rats. Conversely, application of OT/AVP or NMDA increased dendritic branches in slices of P3-P6 rats. Their effects were inhibited by blockade of electri- cal activity, voltage-gated $\mathrm{Ca}^{2+}$ channels, or intracellular $\mathrm{Ca}^{2+}$ mobilization. They were also interdependent because both OT/ AVP and NMDA (but not AMPA) receptor activation were required for increasing the dendritic arbor. Part of this interdependence probably results from a retrograde action of the peptides facilitating glutamate release. Finally, blocking OT/ AVP receptors by in vivo treatment with the peptides antagonists during development decreased spontaneous glutamatergic synaptic activity recorded in young adults. These results show that an interplay between postsynaptic dendritic peptide release and presynaptic glutamate release is involved in the transient increase in dendritic arbor of SON neurons and indicate that OT/AVP are required for normal synaptogenesis of glutamatergic inputs in SON.

Key words: oxytocin; vasopressin; glutamate; NMDA receptor; development; electrical activity; retrograde messenger
The structure of the dendritic arbor is a critical feature determining the number, diversity, and integration of inputs received by a neuron (Koester and O'Leary, 1994). For instance, alteration in the development of the neuronal morphology is likely to affect normal brain function. Reduced dendrites have been related to mental retardation in children (Purpura, 1975), strongly suggesting that neuronal structure and cognitive capacity are closely related. The formation of dendritic arbor and synaptic connections is a highly dynamic process. Neurites bearing postsynaptic elements, such as filopodia, display a high motility during synaptogenesis, whereas synapses are repeatedly formed and eliminated before the mature connection pattern is achieved. Recent in vivo studies in zebra fish and rat have shown that dynamic interactions between filopodia and growth cones occur (Jontes et al., 2000) and that the rate of filopodia motility depends on the level of input activity (Lendvai et al., 2000). Here, we studied the influence of interactions between presynaptic and postsynaptic activities on the dendritic arbor plasticity during synaptogenesis in the supraoptic nucleus (SON).

Hypothalamic SON neurons project to the neurohypophysis in

Received Feb. 26, 2001; revised Sept. 19, 2001; accepted Oct. 19, 2001.

This work was supported in part by Hoechst-Marrion-Roussel Grant 751285/00. We thank N. C. Spitzer for careful reading and correction of this manuscript. This work benefited from fruitful discussions with N. Hussy, G. Alonso, and A. Rabié and their suggestions concerning this manuscript.

Correspondence should be addressed to Michel G. Desarménien, Centre National de la Recherche Scientifique Unité Mixte de Recherche 5101, Biologie des Neurones Endocrines, 141 rue de la Cardonille, 34094 Montpellier Cedex 5, France. E-mail: mgdesa@ccipe.montp.inserm.fr.

Copyright (ㄷ) 2001 Society for Neuroscience $\quad 0270-6474 / 01 / 220265-09 \$ 15.00 / 0$ which they secrete oxytocin or vasopressin (OT/AVP) into the blood circulation. The peptides are also released at dendritic sites and exert an autocontrol on their secreting neurons. During development, the peptides maintain spontaneous electrical activity via a depolarization and a facilitation of their own dendritic release (Chevaleyre et al., 2000). This autocontrol loop is maximum during the second postnatal week (PW2) and is correlated with a transient increase in dendritic branching (Chevaleyre et al., 2001). Meanwhile, functional monoaminergic synapses are formed (Ugrumov, 1992; Nelson et al., 1998), NMDA receptor density transiently increases (Hussy et al., 1997), and glutamatergic postsynaptic potentials appear (Chevaleyre et al., 2001), indicating an intense period of synapse formation. In view of this concomitance of events, we studied the role of dendritic peptide release and its interaction with glutamate released by incoming inputs [the major excitatory neurotransmitter in the SON (van den Pol et al., 1990)] on the morphological plasticity observed in SON neurons. We used in vivo and in vitro experiments to show that NMDA receptor and OT/AVP receptor activation are both needed to increase the dendritic arbor, suggesting that an interplay between presynaptic and postsynaptic activities is required for this plasticity. In addition, the peptides are able to act on presynaptic endings and increase glutamatergic synaptic transmission. Moreover, injection of OT/AVP antagonists during the first two postnatal weeks (PW2) decreased spontaneous synaptic activity in SON slices from young adult rats, suggesting that early activation of OT/AVP receptors indeed plays a determinant role for normal synaptogenesis in SON. 


\section{MATERIALS AND METHODS}

In vivo drug treatment. Some littermates were assigned to receive daily subcutaneous injection of a solution containing either $(+)$-5-methyl10,11-dihydro-5H-dibenzo [a,d] cyclohepten-5,10-imine maleate $(\mathrm{MK} 801)(1 \mathrm{mg} / \mathrm{kg} ; 1 \mu \mathrm{l} / \mathrm{gm})$ or a mixture of OT antagonist and $\mathrm{V}_{1 \mathrm{a}}$ and $\mathrm{V}_{2}$ AVP antagonists $(9,0.5$, and $0.6 \mu \mathrm{g} / \mathrm{kg}$, respectively; $0.7 \mu \mathrm{l} / \mathrm{gm}$ ) from birth to postnatal day 7 (P7) or P8. Others pups of the litter received saline (with $0.36 \%$ DMSO for controls of OT/AVP-treated rats). Pups were killed 14-16 hr later, and the morphology of SON neurons was studied in acute slice (see below). For experiments on synaptic activity, rats were treated daily with saline or OT/AVP antagonists mixture from P3 to P17, and glutamatergic activity was recorded during PW4 (see below).

Slice preparation and drug treatment. Standard methods were used as described previously (Chevaleyre et al., 2000). Briefly, P3-P9 rats were killed by decapitation, and brains were rapidly removed and immersed in a cold oxygenated $\left(95 \% \mathrm{O}_{2}, 5 \% \mathrm{CO}_{2}\right)$ sucrose solution [in mM: 220 sucrose, $2.3 \mathrm{KCl}, 26 \mathrm{NaHCO}_{3}, 2.5 \mathrm{CaCl}_{2}, 10$ glucose, $1.2 \mathrm{KH}_{2} \mathrm{PO}_{4}$, and 1.2 $\mathrm{MgSO}_{4}, \mathrm{pH} 7.4$ (300 mOsm/1)]. A block containing the hypothalamus was dissected out and mounted on a vibratome (Campden Instruments, Loughborough, UK), and a horizontal slice (250- $\mu \mathrm{m}$-thick) was cut. Each half containing one SON was then incubated in oxygenated $\left(95 \% \mathrm{O}_{2}, 5 \%\right.$ $\mathrm{CO}_{2}$ ) artificial CSF (ACSF) [in mM: $110 \mathrm{NaCl}, 1.2 \mathrm{KCl}, 26 \mathrm{NaHCO}_{3}, 2$ $\mathrm{CaCl}_{2}, 10$ glucose, $1.2 \mathrm{KH}_{2} \mathrm{PO}_{4}$, and $2 \mathrm{MgCl}_{2}, \mathrm{pH} 7.4$ (300 mOsm/1)] supplemented with various agents during $3-8 \mathrm{hr}$ at room temperature. A half slice was then transferred into an immersion type recording chamber, under the objective $(40 \times)$ of a conventional microscope and perfused with oxygenated $\left(95 \% \mathrm{O}_{2}, 5 \% \mathrm{CO}_{2}\right) \operatorname{ACSF}\left(34^{\circ} \mathrm{C}\right)$ at a rate of 2 $\mathrm{ml} / \mathrm{min}$, and neurons were dyed with Lucifer yellow (LY).

Morphological analysis. Neurons were loaded with LY (Sigma, St. Quentin Fallavier, France) added into the intrapipette solution [in mM: $135 \mathrm{KMeSO}_{3}, 5 \mathrm{KCl}, 1 \mathrm{CaCl}_{2}, 5$ EGTA-Na, 4 ATP-Mg, 10 HEPES-Na, and $1 \mathrm{LY}, \mathrm{pH} 7.2$ (290 mOsm/1)]. Neurons with large cell body and generally exhibiting an $\alpha$ dendrite (Hatton, 1990), shown previously to synthesize either OT or AVP at all stages of postnatal development (Hussy et al., 1997), were selected in the SON. After establishment of the whole-cell configuration, LY diffusion was facilitated by application of repetitive voltage steps from -60 to $-90 \mathrm{mV}$ during $\sim 30 \mathrm{~min}$. Neuronal shapes were then drawn either directly or after acquisition of $\mathrm{z}$ series images with a PCO camera (Dipsi, Chatillon, France) and Axon Imaging Workbench software (Axon Instruments, Foster City, CA). The number and order of each dendritic branch was measured. To avoid variability between rats, all comparisons were made between the two SON of the same rat incubated during the same time (3-8 hr) in different solutions. Results were pooled only if the experimental conditions for each of the two SONs of each rat were identical. Data are expressed as mean \pm SEM, and significance was evaluated using ANOVA test.

Electrophysiological recordings. Horizontal slices were prepared from P7-P9 or P21-P23 rats, and the neurons were visualized under infrared interference microscopy. Because the number of synapses can increase after slicing (Kirov et al., 1999), care was taken to respect similar delay between slicing and recording for control and treated rats (between 2 and $8 \mathrm{hr})$. Slices were perfused with $\operatorname{ACSF}\left(34^{\circ} \mathrm{C}\right)$ at a rate of $2 \mathrm{ml} / \mathrm{min}$, and neurons were recorded in the whole-cell configuration with 3-5 M $\Omega$ patch pipettes containing the intrapipette solution without LY (see above). EPSCs were recorded and digitized $(6.6 \mathrm{kHz}$; Clampex software; Axon Instruments) and filtered at $5 \mathrm{kHz}$ (eight-pole Bessel filter) for at least $10 \mathrm{~min}$ from a holding potential of $-60 \mathrm{mV}$; series resistance was controlled before and after this collection. EPSCs were analyzed off-line using a home-made routine (Origin; Microcal Software Inc., Northampton, MA) for measurement of frequency and amplitude. Recordings were performed close to the reversal potential of GABA-evoked chloride currents and in the presence of a $\mathrm{GABA}_{\mathrm{A}}$ receptor blocker $(3 \mu \mathrm{M}$ gabazin) in two-thirds of the neurons. No difference was observed in the mean amplitude and frequency between neurons recorded with or without gabazin, indicating that only glutamatergic EPSCs were recorded.

Pharmacological compounds. OT/AVP antagonists consisted of a cocktail of an OT antagonist [100 nM dOVT or Manning compound: $\mathrm{d}\left(\mathrm{CH}_{2}\right)_{5}\left[\mathrm{Tyr}(\mathrm{Me})^{2}, \mathrm{Thr}^{4}, \mathrm{Tyr}_{\mathrm{NH}}{ }_{2}{ }^{9}\right.$; kindly provided by Dr C. Barberis, Montpellier, France], a $\mathrm{V}_{1 \mathrm{a}}$ receptor antagonist [10 nM SR49059: $(2 S) 1[(2 R, 3 S)-(5$-chloro-3-(2-chlorophenyl)-1-(3,4-dimethoxybenzenesulfonyl))-3-hydroxy-2,3-dihydro-1H-indole-2-carbonyl]-pyrrolidine-2carboxamide], and a $\mathrm{V}_{2}$ antagonist [10 nM SR121463A: 1-[4-(N-tertbutylcarbamoyl)-2-metoxybenzene sulfonyl]-5-ethoxy-3-spiro-[4-(2morpholino ethoxy) cyclohexane]-indol-2-one, furamate; equatorial isomer] (both provided by Sanofi Synthelabo Recherche, Toulouse, France). OT and AVP were purchased from Calbiochem-Novabiochem (La Jolla, CA). CNQX and MK801 were purchased from Research Biochemicals (Sigma). Thapsigargin, $\omega$-conotoxin-GVIA, and $\omega$-agatoxin-IVA were purchased from Alomone Labs (Jerusalem, Israel). CNQX, NMDA, TTX, nicardipin, and all salts were purchased from Sigma.

\section{RESULTS}

SON neurons from hypothalamic slices were injected with Lucifer yellow through a patch-clamp pipette, drawn after $30 \mathrm{~min}$ diffusion of the dye, and the number of dendritic branches was counted. Previous study has shown that the number of branches increases in SON neurons at the beginning of PW2 and then decreases during PW2-PW3, leading to bipolar or tripolar neurons with a few branching (Chevaleyre et al., 2001). Here, the mechanisms involved in the increase in dendritic arbor were studied in 414 neurons dyed after different experimental conditions during two developmental periods: between P3 and P6, the period preceding the normal increase in dendritic branches, and between P7 and P9, the period of maximal dendritic arbor. The mean number of dendritic branches per neuron in control condition was $12.6 \pm 0.6$ at P3-P6 $(n=53)$ and $16.2 \pm 0.7$ at P7-p9 $(n=39 ; p<0.01)$.

\section{Role of OT/AVP in shaping the dendritic arbor}

Rat pups were injected daily with OT/AVP antagonists from birth to P6 or P7. The morphology of the dendrites was studied 14-16 hr later. A decrease in the dendritic arbor was observed in OT/AVP antagonist-treated rats compared with saline-treated rats (Fig. 1a). The number of primary dendrites was not affected (Fig. $1 b)$, but the total number of dendritic branches was reduced by $36 \%$ (Fig. 1c). To analyze the underlying mechanisms, experiments with acute hypothalamic slices were performed. For each slice, one-half containing one SON was incubated 3-8 hr with OT/AVP antagonists, and the dendritic arbor was compared with that of neurons of the other SON incubated in control condition. Incubation with OT/AVP antagonists (100 and $10 \mathrm{~nm}$ for OT and AVP antagonists, respectively) significantly decreased the large dendritic arbor of $\mathrm{P} 7-\mathrm{P} 9$ rats (37\% decrease) (Fig. 1d,e), whereas it had only a slight effect before the normal increase in P3-P6 rats (12\% decrease) (Fig. 1f, g). Conversely, incubation with OT/AVP (each at $100 \mathrm{nM}$ ) increased the number of branches (Fig. 2a). The effects of OT/AVP were small and nonsignificant at P7-P9 (15\% increase) (Fig. 2d,e) but significant at P3-P6 (53\% increase) (Fig. $2 f, g)$. Again, the number of primary dendrites was not changed by the various treatments, with only higher-order dendrites being affected. These results show that the peptide receptors and growth mechanisms are already functional at P3-P6 and that endogenous peptides are involved in the increase and maintenance of the dendritic arbor at P7-P9.

\section{Role of NMDA receptor activation in shaping the dendritic arbor}

Because glutamatergic EPSPs appear and somatic NMDA receptors transiently increase during PW2 in SON neurons, we tested the role of NMDA in dendritic plasticity. In vivo daily injection of the NMDA antagonist MK801 from P0 to P6 resulted in a significant reduction (27\%) in the number of dendritic branches observed at P7 (Fig. 3a-c). Incubation of a slice with MK801 (1 $\mu \mathrm{M}$ ) did not change the number of primary dendrites but decreased the dendritic branches in a manner similar to OT/AVP antagonists. The decrease was important at P7-P9 (34\% decrease) (Fig. 3d,e) and small at P3-P6 (6\% decrease) (Fig. 3f, g). Conversely, incubation with NMDA $(1 \mu \mathrm{M})$ increased the den- 
a

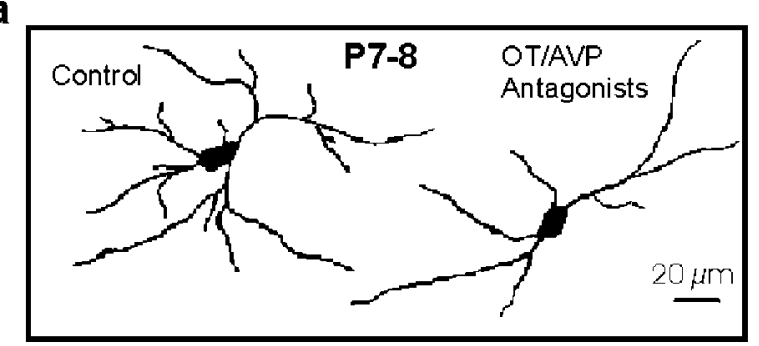

b

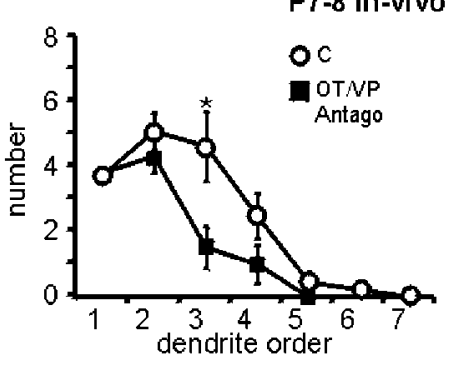

d

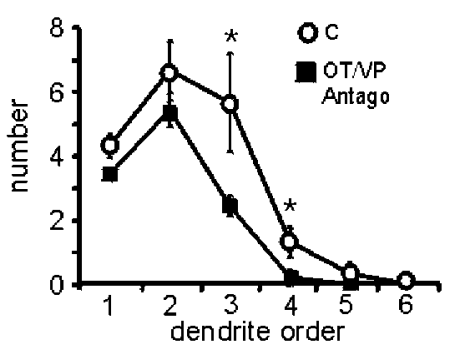

f

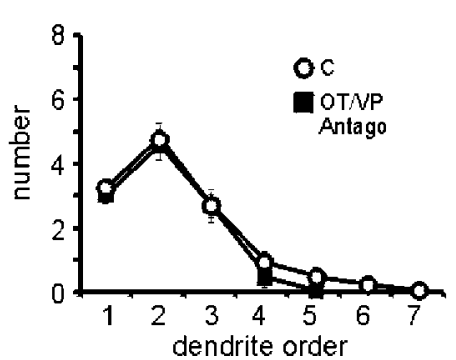

C

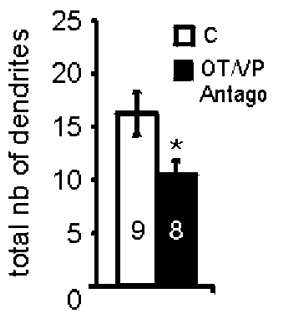

e

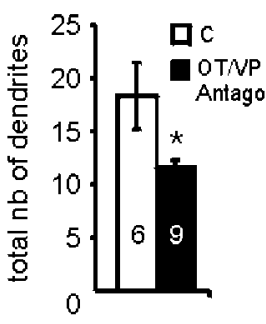

g

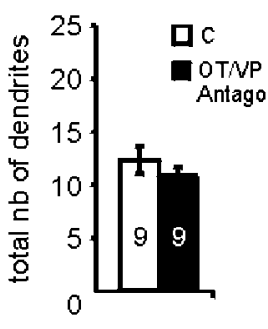

Figure 1. Endogenous OT/AVP are required for dendritic arbor maintenance. $a$, Example of representative SON neurons dyed at P7 from a control and an OT/AVP antagonist-treated rat. $b$, Mean number of dendrites versus dendrite order for neurons dyed from P7-P8 control $(C$; open circles) or OT/AVP antagonist (OT/VP Antago)-treated rats ( filled squares), indicating that mostly third- and higher-order dendrites were affected. $c$, Neurons of OT/AVP antagonist-treated rats displayed an important decrease in their total number of dendrites compared with control ones. $d-g$, In vitro incubation of the slices in OT/AVP antagonists led at P7-P9 to a similar decrease in the dendritic arbor compared with the in vivo treatment $(d, e)$ but had almost no effect at $\mathrm{P} 3-\mathrm{P} 6(f, g)$. Note that, at $\mathrm{P} 7-\mathrm{P} 9$, antagonists reduced the number of dendritic branches to values similar to that of P3-P6 controls. The number of neurons is indicated in each bar $\left({ }^{*} p<0.05\right.$; ANOVA).

dritic arbor (Fig. 4a). As for OT/AVP, the NMDA effect was marked at P3-P6 (32\% increase) (Fig. 4b,c) and smaller at P7-P9 (12\% increase) (Fig. 4d,e). These results show that NMDA receptor activation by endogenous glutamate is necessary for the increase and maintenance of the dendritic arbor.

Role of electrical activity in shaping the dendritic arbor Because electrical activity is increased by OT/AVP and NMDA, its role in dendritic plasticity was investigated using TTX $(0.3$
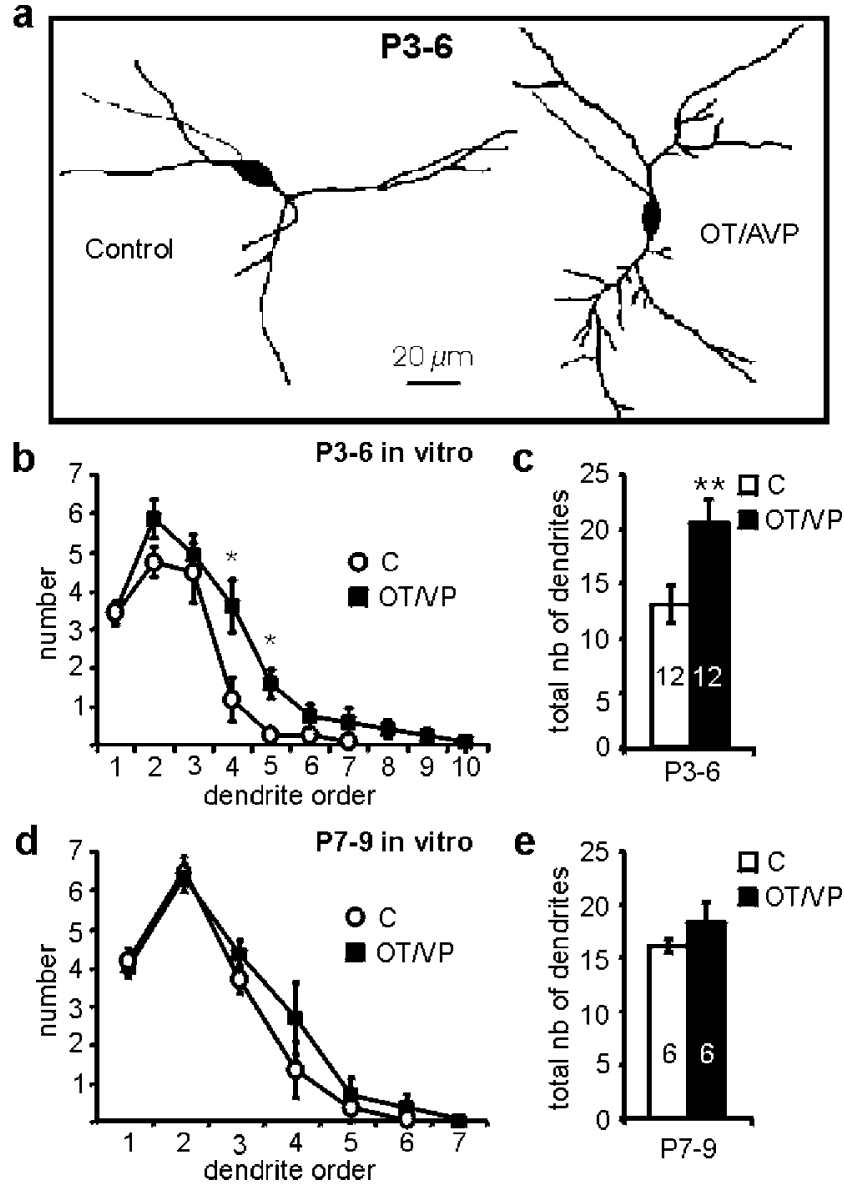

Figure 2. OT/AVP increase the number of dendritic branches. $a$, Example of representative SON neurons dyed at P4-P5 in control condition or after in vitro incubation in OT/AVP showing an increase in the number of branches. $b$, Mean number of dendrites versus the dendrite order at P3-P6 showing no effect of OT/AVP (OT/VP; filled squares) on primary dendrites and a significant increase in fourth- and fifth-order dendritic branches compared with control neurons $(C$; open circles $)$. This resulted in an important increase in the total number of dendritic branches $(c)$. At P3-P6, however, OT/AVP application only slightly increased fourth-order dendrites $(d)$ and had no significant effect on the total number of dendrites $(e)$. The number of neurons is indicated in each $\operatorname{bar}\left({ }^{* *} p<0.01\right.$; ANOVA).

$\mu \mathrm{M})$. At P7-P9, a decrease in the number of branches was observed when TTX $(0.3 \mu \mathrm{M})$ was applied in the presence of OT/AVP and NMDA to compensate for a putative decrease in endogenous peptides and glutamate release (Fig. 5a). Conversely, application of OT/AVP and NMDA did not increase the dendritic arbor at P3-P6 in the presence of TTX, even when the voltage-dependent block of NMDA receptors was relieved by omitting $\mathrm{Mg}^{2+}$ from the medium (Fig. 5b). Electrical activity seems, therefore, to be involved in the increase in dendritic branches. However, activity could act indirectly, via an increase in endogenous peptides release. This would imply that the amount of exogenous peptides was not sufficient to increase branching and that additional endogenous peptides release was needed. This seems unlikely because increasing the peptides concentration by a factor 10 or 100 did not counteract the effect of TTX at P3-P6 (106 and 97\% of TTX alone with 1 and $10 \mu \mathrm{M}$ OT/AVP, respectively; $n=7$ and 8; data not shown). However, electrical activity is not sufficient to increase the dendritic arbor because a high $\mathrm{K}^{+}$ solution, able to increase the number of dendritic branches at 
a

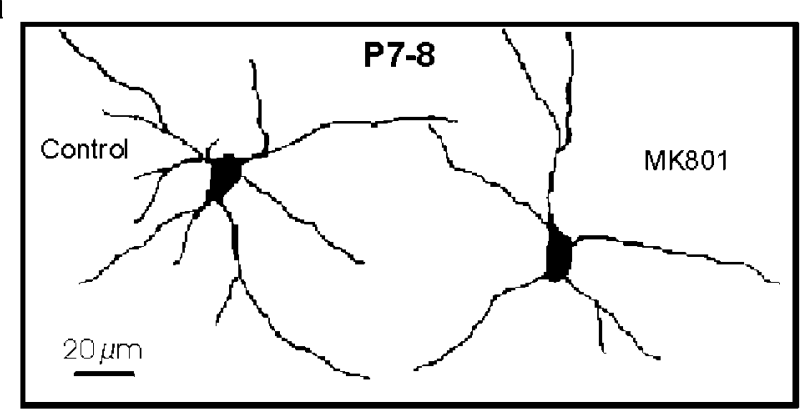

b

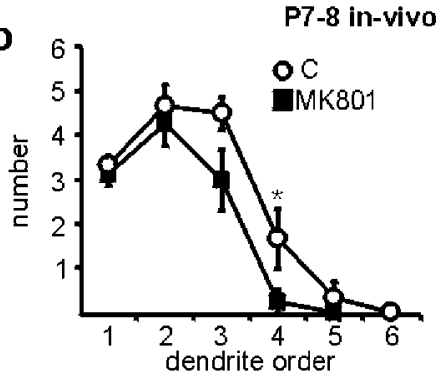

d
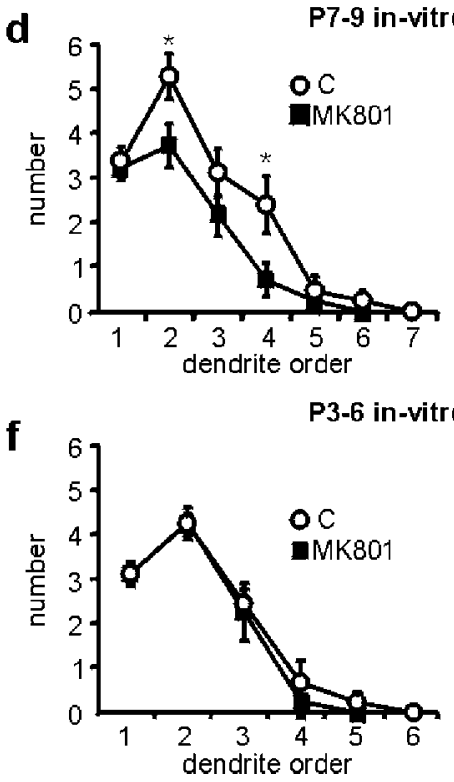
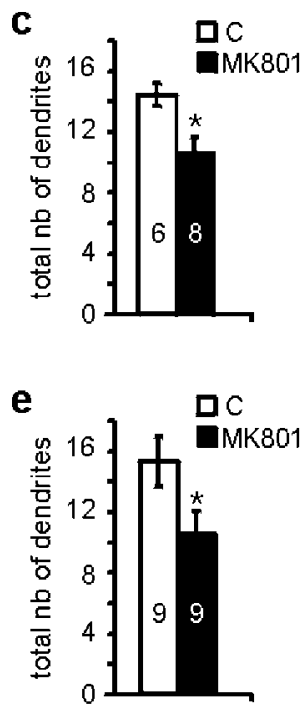

\section{g}

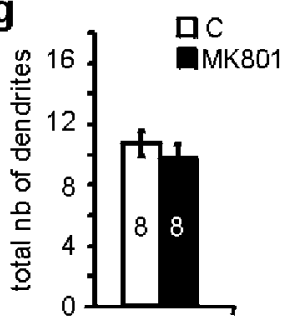

Figure 3. Natural activation of NMDA receptor is necessary for dendritic arbor maintenance. $a$, Example of representative SON neurons at P7, showing a decrease in the number of branches after incubation in MK801. $b$, Mean number of dendritic branches versus the dendrite order for neurons dyed from control ( $C$; open circles) or MK801-treated rats (MK801; filled squares) showing a decrease in third- and fourth-order dendrites. $c$, The total number of dendritic branches was significantly decreased in MK801-treated rats. $d-g$, In vitro experiments showing an important decrease in branching by MK801 in slices of P7-P9 rats $(d, e)$ but not in slices of $\mathrm{P} 3-\mathrm{P} 6$ rats $(f, g)$. The number of neurons is indicated in each $\operatorname{bar}\left({ }^{*} p<0.05\right.$; ANOVA).

P3-P6, had no effect when OT/AVP and NMDA receptors were blocked (Fig. $5 c$ ). Altogether, these results suggest an hypothesis in which electrical activity is involved in the increase in the dendritic arbor independently of increasing endogenous peptides release but is not a unique downstream consequence in OT/AVP and NMDA action on dendrites.

\section{Role of $\mathrm{Ca}^{2+}$ in shaping the dendritic arbor}

Whatever its precise implication in the process of increasing the dendritic arbor, electrical activity could act by triggering $\mathrm{Ca}^{2+}$
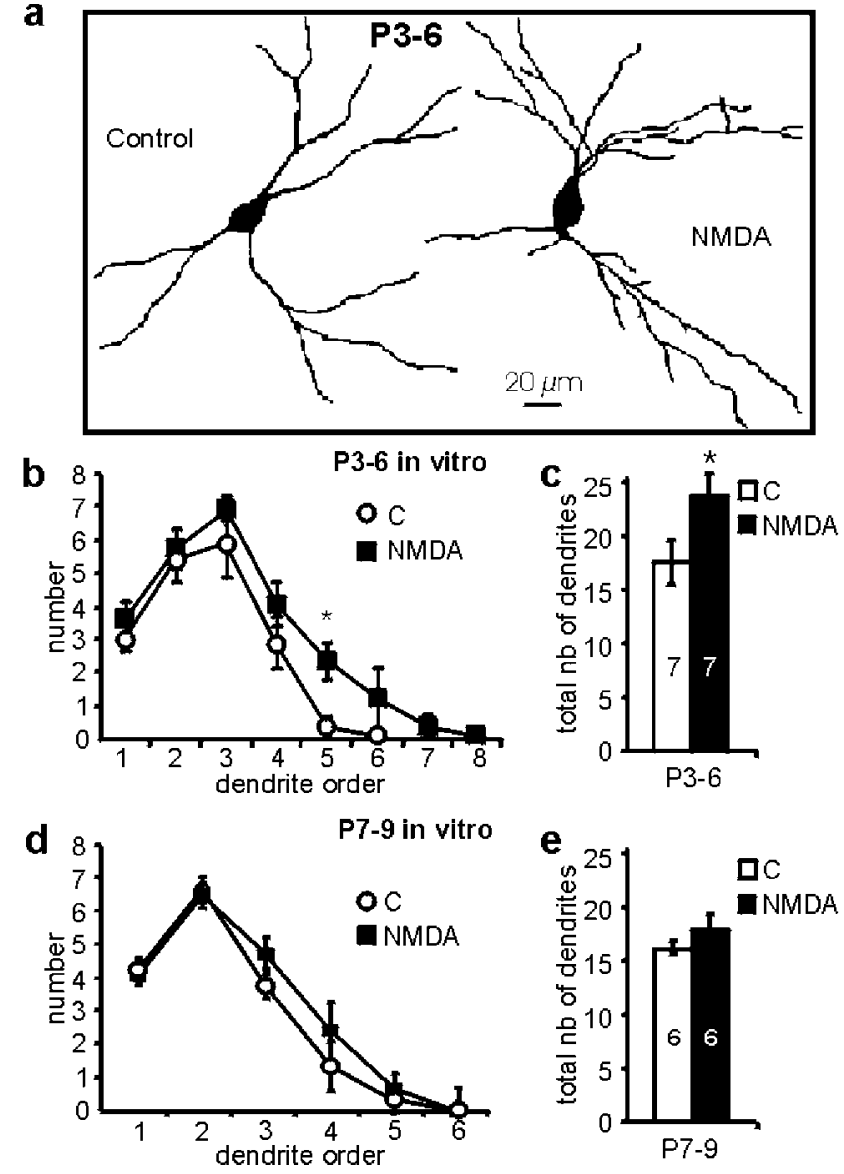

Figure 4. NMDA increases the number of dendritic branches. $a$, Example of representative neurons dyed at P4 indicating an increase in small branches after incubation in NMDA. $b$, The mean number of dendrites by order revealed that, at P3-P6, NMDA (filled squares) had no effect on first- and second-order dendrites but increased significantly fifth-order dendrites compared with controls ( $C$; open circles). This resulted in a significant increase in the total number of dendritic branches at P3-P6 (c). Conversely, at P7-P9, NMDA had only slight effects on third- and fourth-order dendrites $(d)$ and did not change significantly the total number of dendrites $(e)$. The number of neurons is indicated in each bar $\left({ }^{*} p<0.05 ;\right.$ ANOVA).

influx via voltage-gated $\mathrm{Ca}^{2+}$ channels (VGCCs). At P3-P6, in the presence of toxins for $\mathrm{L}, \mathrm{N}$, and $\mathrm{P} / \mathrm{Q}$ channels $(3 \mu \mathrm{M}$ nicardipin, $500 \mathrm{~nm} \omega$-conotoxin-GVIA, and $200 \mathrm{~nm} \omega$-agatoxin-IVA, respectively), OT/AVP, NMDA, and high $\mathrm{K}^{+}$had no effect on the dendritic arbor (Fig. 5c). Because OT/AVP are known to mobilize intracellular $\mathrm{Ca}^{2+}$ in adults, stores were depleted with thapsigargin $(0.2 \mu \mathrm{M})$, a blocker of the reticulum $\mathrm{Ca}^{2+}$-ATPase. In the presence of thapsigargin, which did not change the number of dendritic branches compared with control $(n=5$ and 4 , respectively; data not shown), OT/AVP and NMDA were unable to increase the dendritic arbor at P3-P6 (Fig. 5d). These results suggest that both $\mathrm{Ca}^{2+}$ influx via VGCC and $\mathrm{Ca}^{2+}$ release from intracellular stores are involved in the increase in the dendritic arbor.

\section{Interdependence of OT/AVP and NMDA effects}

To study whether OT/AVP and NMDA act on separated pathways, OT/AVP were applied at P3-P6 in the presence of MK801. Under this condition, OT/AVP were unable to increase the number of dendritic branches (Fig. 6a). However, because electrical 


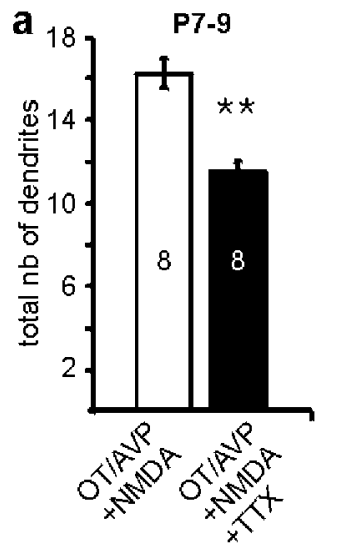

C $\quad \star \star \star$ P3-6
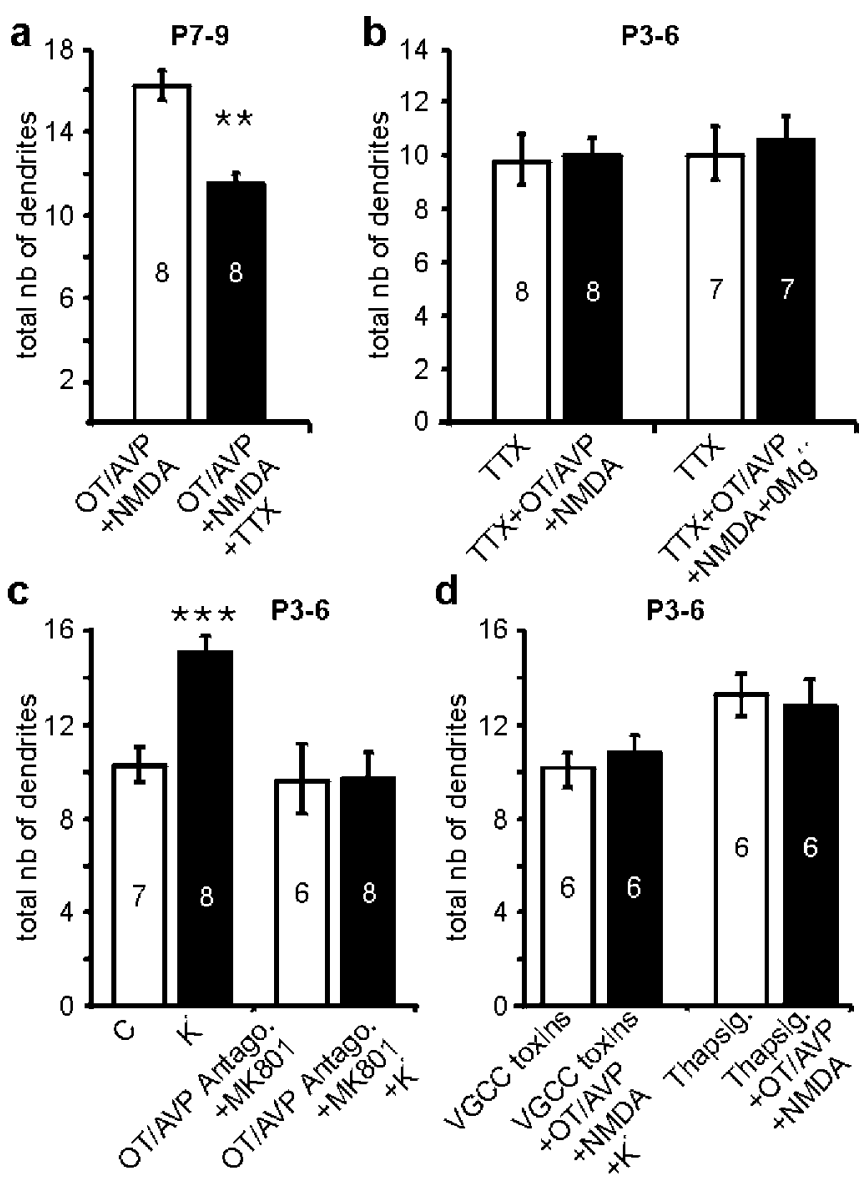

Figure 5. Electrical activity, voltage-gated $\mathrm{Ca}^{2+}$ channels, and intracellular $\mathrm{Ca}^{2+}$ stores are involved in the effect of OT/AVP and NMDA. $a$, Suppression of electrical activity by TTX at P7-P9 decreased the number of dendritic branches, even when OT/AVP and NMDA were added to compensate for the decrease in endogenous release induced by TTX. All other experiments were performed at P3-P6. $b$, In the presence of TTX, OT/AVP and NMDA were unable to increase the number of dendritic branches, even when $\mathrm{Mg}^{2+}$ was removed to increase NMDA receptor activation. $c$, Application of $12.5 \mathrm{mM} \mathrm{K}^{+}$increased the total number of dendritic branches. However, when applied in the presence of OT/AVP antagonists (OT/AVP Antago.) and M K801, the high- $\mathrm{K}^{+}$solution had no effect on the dendritic arbor, indicating that electrical activity alone was not effective. $d$, The blockade of $\mathrm{L}-, \mathrm{N}-$, and $\mathrm{P} / \mathrm{Q}$-type voltage-gated $\mathrm{Ca}^{2+}$ channels by a cocktail of toxins (VGCC toxins) disrupted the effects of OT/AVP and NMDA, even when high $\mathrm{K}^{+}$was added. The increase in the dendritic arbor also depends on mobilization of intracellular $\mathrm{Ca}^{2+}$ stores because OT/AVP and NMDA were ineffective in the presence of thapsigargin (Thapsig.). The number of neurons is indicated in each $\operatorname{bar}\left({ }^{*} p<\right.$ 0.05 ; ANOVA).

activity appears to be involved in dendritic plasticity and could have been reduced by MK801, the same experiment was performed in the presence of high $\mathrm{K}^{+}$. Under this condition, OT/ AVP were still unable to increase the dendritic arbor (Fig. 6a). This effect was specific for NMDA receptor activation because OT/AVP increased the number of dendritic branches in the presence of CNQX $(50 \mu \mathrm{M})$, a blocker of AMPA/KA receptors (Fig. 6a), and CNQX alone did not decrease the number of dendritic branches when compared with controls at P7-P9 $(n=4$ and 4; data not shown). Conversely, when applied with OT/AVP antagonists, NMDA had no effect on the dendritic branches, even in a high-K $\mathrm{K}^{+}$medium (Fig. $6 b$ ). These results show that activation of both NMDA receptors and OT/AVP receptors are needed for increasing the dendritic arbor.
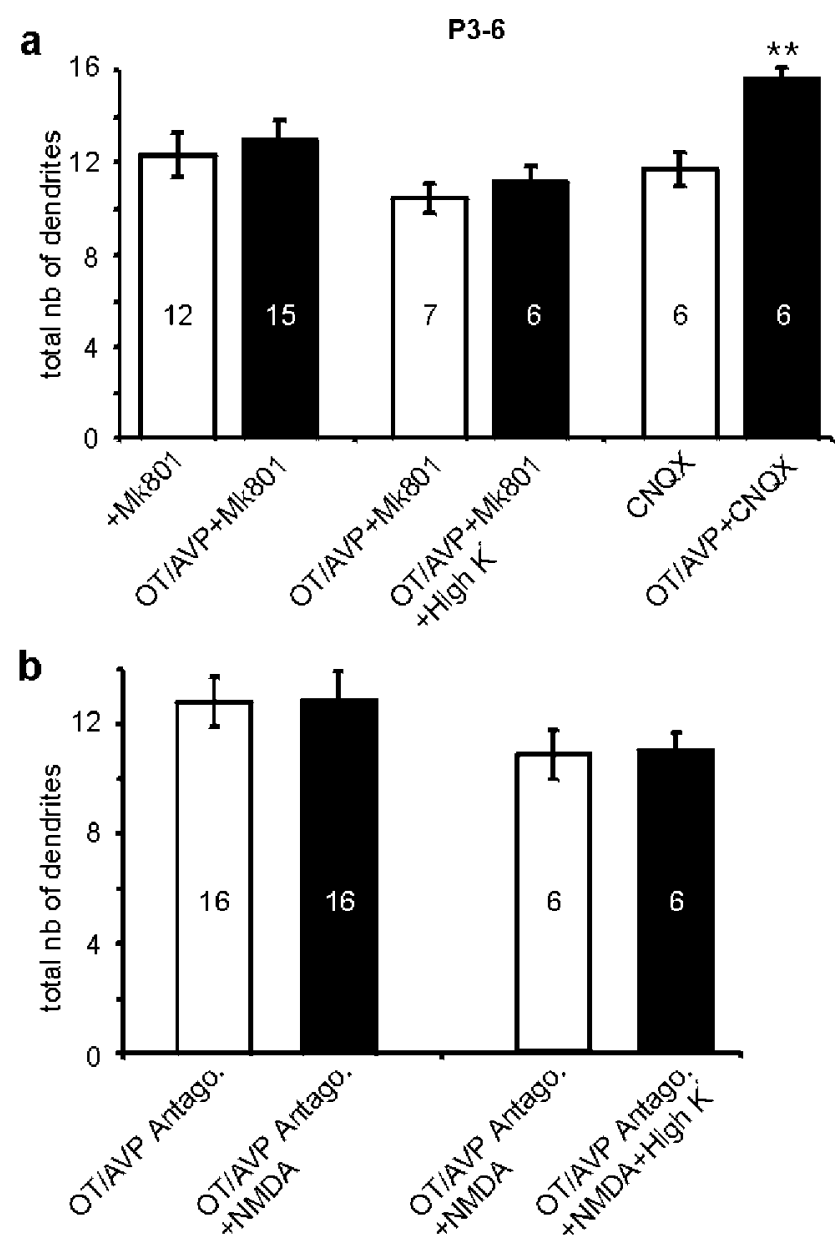

Figure 6. OT/AVP and NMDA effects are interdependent. $a$, Application of OT/AVP had no effect on the number of dendritic branches when NMDA receptors were blocked by MK801, even in the presence of high $\mathrm{K}^{+}$. Conversely, AMPA receptor blockade by CNQX did not suppress the increase in the number of dendritic branches induced by OT/AVP. $b$, Activation of NMDA receptors did not increase the dendritic arbor in the presence of OT/AVP antagonists (OT/AVP Antago.), even in the presence of $12.5 \mathrm{mM} \mathrm{K}^{+}$. The number of neurons is indicated in each bar $(* * p<$ 0.01 ; ANOVA).

\section{Acute effect of OT/AVP on synaptic activity}

Because a presynaptic action of OT on glutamatergic endings has been described in adults, we tested whether part of the interdependence may result from a facilitatory effect of the peptides on glutamate release. The spontaneous glutamatergic synaptic activity was recorded in slices of P7-P9 rats, a period just after the appearance of the first EPSPs. Experiments were initially performed with the peptides at $1 \mu \mathrm{M}$ because no presynaptic effects were observed in adults below this concentration (Kombian et al., 1997). In 10 of 14 neurons, OT/AVP induced a marked increase in EPSC frequency (Fig. $7 a-c$ ). Among the four insensitive neurons, three displayed the highest mean EPSC frequency, which was decreased (Fig. 7d) by OT/AVP antagonists. This shows that, in some neurons, endogenous OT/AVP exert a maximal increase in synaptic activity, which cannot be further enhanced by exogenous peptides. The peptides are also efficient at $0.1 \mu \mathrm{M}$ but with smaller effects ( $42 \%$ of the effect of $1 \mu \mathrm{M}$ on EPSP frequency; $n=$ $3)$. In four neurons in which OT/AVP induced the strongest increase in frequency, a $30-100 \%$ increase in EPSC amplitude was also observed during OT/AVP application (Fig. 7e). These 


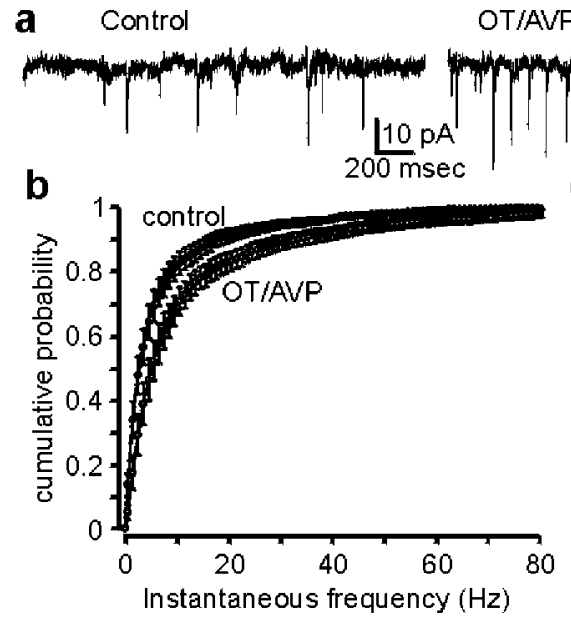

OT/AVP

d

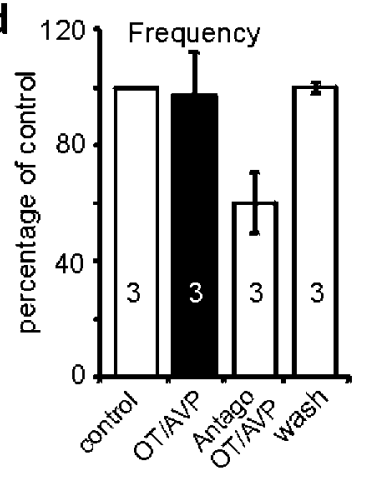

$\mathbf{f}$

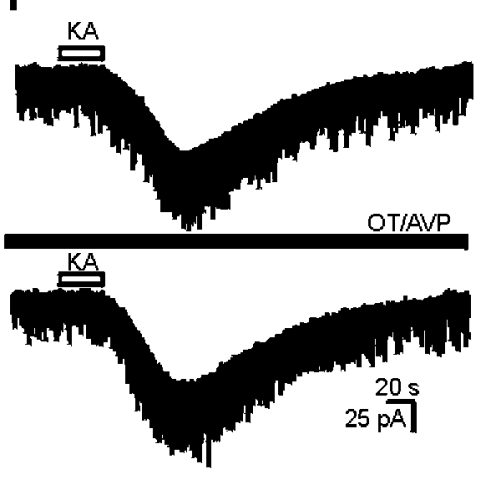

e

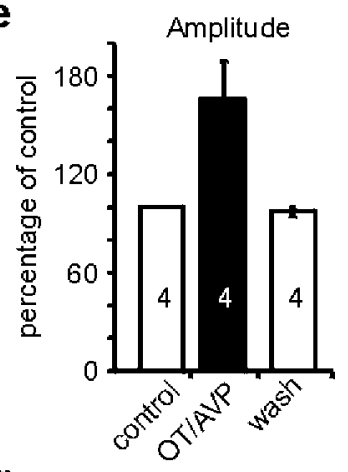

g

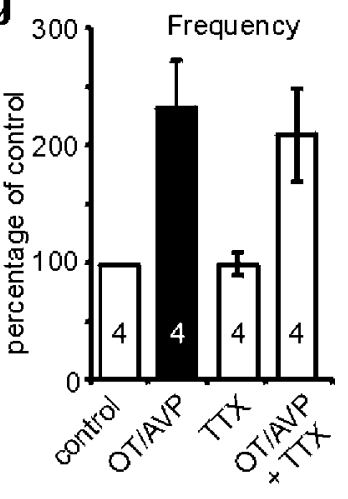

Figure 7. OT/AVP act presynaptically to increase glutamatergic transmission. $a$, Spontaneous EPSCs recorded at $-60 \mathrm{mV}$, in the presence of the $\mathrm{GABA}_{\mathrm{A}}$ receptor antagonist GABAzine $(3 \mu \mathrm{M})$, in a neuron of a P8 rat in control condition and during OT/AVP application. $b$, Graph of the mean cumulative probability showing an increase in EPSCs frequency during OT/AVP application (Kolmogorov-Smirnov test; $p<0.0001$ ). $c$, Mean effects of OT/AVP on EPSC frequency for the 10 neurons in which the frequency was increased by the peptides. $d$, Effect of OT/AVP antagonists in three neurons not affected by OT/AVP, demonstrating the implication of endogenous peptides on EPSCs frequency. $e$, In four neurons in which OT/AVP induced the strongest increase in EPSCs frequency, an increase in EPSC amplitude was also observed. In these neurons, the effect on EPSC amplitude appears to be presynaptic because OT/AVP did not affect kainate $(K A ; 25 \mu \mathrm{M})$-evoked current $(f) . g$, OT/AVP did not act by increasing electrical activity of presynaptic neurons because their effect on EPSC frequency was not affected by TTX $(0.3 \mu \mathrm{M})$.

results suggest a presynaptic action of the peptides in neurons in which EPSC amplitude was not affected. In the other neurons, a postsynaptic increase in EPSC amplitude may also be responsible for the increase in frequency. To test this possibility, and because

the NMDA receptor antagonist MK801 (1 $\mu \mathrm{M})$ did not affect spontaneous or OT/AVP-evoked EPSCs $(n=3$; data not shown), the current evoked by application of kainate $(25 \mu \mathrm{M}, 30 \mathrm{sec})$ was studied in control condition and under OT/AVP exposure. In neurons in which EPSC amplitude was increased by the peptides, the presence of OT/AVP affected neither the amplitude nor the kinetics of kainate-evoked current $(n=3)$ (Fig. $7 f)$. This indicates that, even in cells in which EPSC amplitude was increased, OT/AVP increased synaptic transmission by acting at presynaptic sites. To exclude the possibility that OT/AVP acted on the cell body of afferent neurons to increase electrical activity and synaptic transmission, the peptides were applied in the presence of TTX. TTX $(0.3 \mu \mathrm{M})$ alone had no effect on spontaneous EPSC frequency and did not affect the increase in frequency induced by OT/AVP $(n=4)$ (Fig. $7 g)$. Together, these results show that, during synaptogenesis, dendritically released OT/AV P are able to act on presynaptic endings and increase glutamatergic transmission.

\section{Putative role of OT/AVP on synaptogenesis}

The role of OT/AVP in increasing the dendritic arbor and glutamate release suggests an important function of these peptides in glutamatergic synapse establishment. To test this hypothesis, OT/AVP receptors were blocked by daily injection of OT/AVP antagonists during a period expected to overlap the one of glutamatergic synapse establishment (P3-P17). The spontaneous glutamatergic synaptic activity was then recorded at P21-P23 in acute horizontal slices of saline- or antagonists-treated rats (Fig. $8 a$ ). This treatment had no effect on the amplitude of glutamatergic EPSCs (Fig. 8b,c) but significantly reduced their frequency (Fig. $8 d, e$ ). The most straightforward explanation is that the number of synapses or the neurotransmitter release probability was decreased. This result supports the idea that OT/AVP receptors indeed play an important role in synaptogenesis of glutamatergic inputs.

\section{DISCUSSION}

This study shows that the transient increase in the dendritic arbor of developing SON neurons is supported by a coordinated action of OT/AVP released by the dendrites themselves and glutamate probably released by incoming afferents. However, some glial cells are known to release glutamate (Parpura and Haydon, 2000), and this possibility cannot be excluded. The action of these transmitters probably requires electrical activity, influx of calcium through voltage-activated channels, and mobilization of intracellular stores. The peptides are also able to act on presynaptic endings and increase glutamate release, and they are probably required for normal synaptogenesis, because chronic OT/AVP receptor blockage during development decreases the frequency of spontaneous synaptic EPSCs in young adults.

\section{Events involved in the increase in the dendritic arbor}

The transient increase in dendritic arbor of SON neurons appears to be a complex phenomenon (Fig. 9). The peptides OT/AVP, released at dendritic sites, play a determinant role in this plasticity. Application of exogenous peptides had maximal effects at P3-P6, whereas endogenous peptides are mostly involved at P7$\mathrm{P} 9$, suggesting that endogenous OT/AVP release is low at P3-P6, whereas receptors and mechanisms involved in dendritic growth are already present. Although the roles of OT and AVP were not studied separately, it is probable that they act in a similar manner on their respective neurons. Both peptides act specifically on their 
a

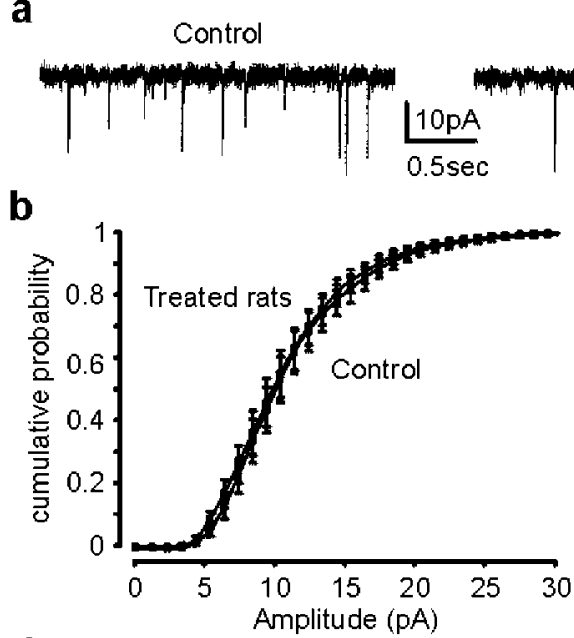

d

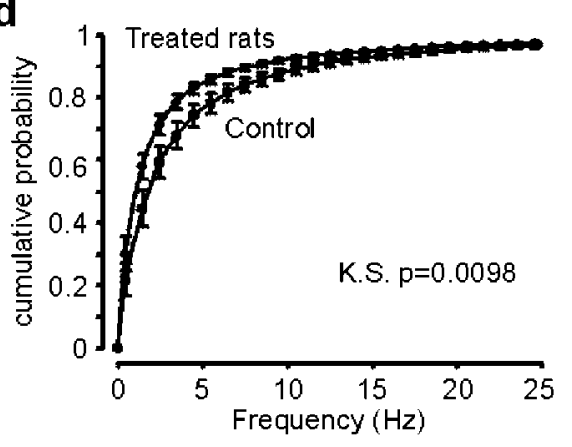

C

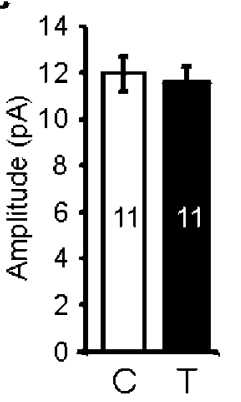

e

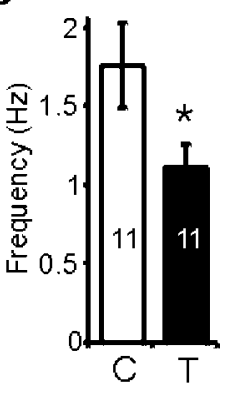

Figure 8. OT/AVP antagonists treatment during development decreases spontaneous glutamatergic synaptic activity in young adults. $a$, Spontaneous EPSCs recorded at $-60 \mathrm{mV}$ in the presence of gabazin in neurons of a P23 control rat and a P23 OT/AVP antagonists-treated rat. $b$, Graph of the mean cumulative probability for the amplitude of the EPSCs showing that the curves in control and treated rats are superposed. $c$, The absence of effect was confirmed by the mean amplitude for all EPSCs that was not affected in treated rats $(T)$ compared with control $(C)$. Conversely, the cumulative probability curves for the instantaneous frequency were significantly different between control and treated rats $(d)$ [KolmogorovSmirnov test $(K . S.) ; p<0.0098]$, as well as the mean frequency of all EPSC $(e)(n=11$ in both conditions; * $p<0.05$; ANOVA $)$.

secreting neurons with similar depolarizing actions and facilitatory effects on their own release during development (Chevaleyre et al., 2000), and both lead to mobilization of intracellular $\mathrm{Ca}^{2+}$ in adult neurons (Dayanithi et al., 2000). These results support the hypothesis that OT and AVP may be directly involved in the dendritic morphology changes (Stern and Armstrong, 1998) and ultrastructural reorganization (Theodosis et al., 1986) observed in mature SON during lactation.

Another major component involved in the control of dendritic arbor is NMDA receptor activation by glutamate release. NMDA receptors regulate dendritic growth during development of neurons in Xenopus (Rajan and Cline, 1998; Li et al., 2000), chick (Wong et al., 2000), mouse (Inglis et al., 1998), rat (Kalb, 1994), and cat (Bodnarenko and Chalupa, 1993) and may do so by activating members of the Rho family GTPases ( $\mathrm{Li}$ et al., 2000; Wong et al., 2000). In the SON, NMDA and MK801 had similar effects as OT/AVP and their antagonists, respectively. NMDA was mostly effective at P3-P6, whereas MK801 had a significant effect only at P7-P9. Glutamate action is probably mediated by the $\mathrm{Ca}^{2+}$ influx evoked by NMDA receptor activation because blockade of AMPA/KA receptors, generally non- $\mathrm{Ca}^{2+}$ perme-

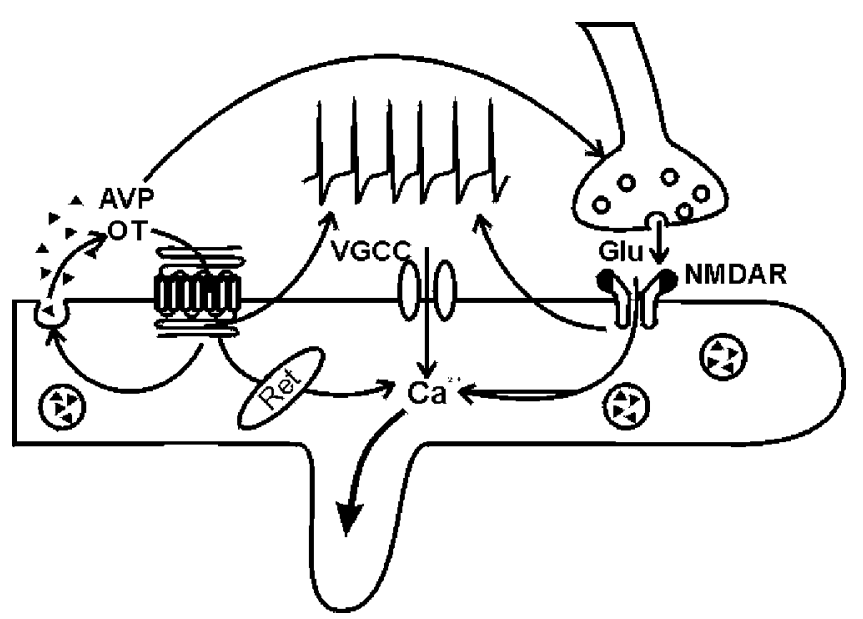

Figure 9. Hypothetical model of the OT/AVP and NMDA action on dendritic arbor. OT/AVP (black triangles) are released at a dendritic level and increase electrical activity and their own dendritic release, mostly during the second postnatal week. This autocontrol loop is involved in the transient increase in dendritic arbor, possibly by increasing intracellular $\mathrm{Ca}^{2+}$ stores mobilization. NMDA receptor activation is also involved in the increase in dendritic arbor, probably by promoting $\mathrm{Ca}^{2+}$ influx. Electrical activity seems also to be involved in increasing the dendritic arbor, independently of increasing endogenous peptides release, and may act by triggering $\mathrm{Ca}^{2+}$ influx via voltage-gated $\mathrm{Ca}^{2+}$ channels. All of these events appear to be implicated in the increase in the number of dendritic branches, showing that presynaptic glutamate release and postsynaptic OT/AVP release are needed to increase the dendritic arbor. The peptides are also able to act on presynaptic endings and increase glutamate release. When OT/AVP receptors are blocked during development, the spontaneous glutamatergic synaptic activity is reduced in young adults, indicating a decrease in the number of synapses or in the probability of spontaneous glutamate release. Another hypothesis is that both OT/AVP and NMDA receptor activation are needed for increasing vesicle exocytosis that releases other molecules than OT/AVP involved in the increase in dendritic branches. Glu, Glutamate; NMDAR, NMDA receptors; Ret, reticulum.

able, did not prevent the increase in the number of dendritic branches.

Electrical activity also appears to be involved in dendritic plasticity. TTX prevented OT/AVP and NMDA actions on the dendritic arbor at P3-P6 and decreased the number of branches at P7-P9. TTX effects are probably not a consequence of a decrease in glutamate release because spontaneous and OT/AVP-evoked EPSCs were not affected by TTX. TTX may have decreased endogenous peptides release, but increasing exogenous peptides concentration by a factor up to 100 did not restore the increase in dendritic branches. Although we cannot be sure that even high concentration of exogenous peptides efficiently counteract a putative decrease in endogenous release, this suggests that electrical activity is involved in increasing the dendritic arbor, independently of increasing endogenous peptides release. However, electrical activity alone is not sufficient because the high- $\mathrm{K}^{+}$-induced increase in dendritic branches was prevented when OT/AVP and NMDA receptors were blocked. One putative role for activity could be to increase $\mathrm{Ca}^{2+}$ influx via VGCCs.

Because activation of both OT/AVP and NMDA receptors is required for increasing dendritic arbor, our results indicate that an interplay between glutamate release and dendritic peptide release is involved in this plasticity. It is not known whether the retrograde action of the peptides is required in this process, but it could explain part of the interdependence between OT/AVP and NMDA actions. However, because dendrites display a rapid 
rate of branch addition and retraction during development, an important issue that remains to be elucidated is whether OT/AVP and NMDA acted by increasing dendritic extension or by decreasing dendritic retraction.

\section{Retrograde action of OT/AVP and roles in synaptogenesis}

Just after the appearance of the first EPSPs at the end of PW1 (Chevaleyre et al., 2001), EPSC frequency was increased by OT/AVP or decreased by their antagonists in almost all neurons tested. Because EPSC amplitude was affected in only some of these neurons and kainate-induced currents were not affected by OT/AVP, the peptides probably acted at a presynaptic site. The increase in amplitude sometime accompanying frequency enhancement may result from intraterminal $\mathrm{Ca}^{2+}$ store mobilization after OT/AVP application, leading to concomitant exocytosis of several vesicles within a release site or between several release sites of the same terminal. Such shared synapses have been described in adult SON and increase in number during physiological plasticity (Theodosis et al., 1995, 1998; Hatton, 1997). A modulatory action of OT and AVP on glutamatergic transmission has been described previously in adult SON (Kombian et al., 1997, 2000). However, both peptides acted by decreasing synaptic transmission in adults, revealing that the effect of OT/AVP on glutamatergic synaptic activity undergoes a developmental switch, from facilitatory to inhibitory. By increasing the dendritic arbor and glutamate release, the peptides may act at different steps of synaptogenesis. If OT/AVP receptors are already expressed before contact between presynaptic and postsynaptic elements, the peptides may either exert an attractive effect or act as a stop signal on growth cones. An indirect action by allowing a transient increase in the dendritic arbor is also expected. Dendritic extensions display a high mobility during synaptogenesis and play an important role in this process (Cline, 2001). Whether or not synapses are formed on the transient dendritic branches remains to be determined. However, transient dendrites may favor interactions with incoming axons, allowing them to form synapses on stable dendrites. Finally, the peptides, by increasing synaptic strength, may favor the maintenance of synaptic contact. Neurotrophins or membrane-permeant molecules, such as arachidonic acid or nitric oxide, have been shown to act as retrograde messengers during synaptic plasticity and synaptogenesis (Fitzsimonds and Poo, 1998). Our results indicate that dendritic neuropeptide release also has a retrograde effect on glutamatergic endings during synaptogenesis. Whatever the exact mode of action of OT/AVP, the fact that chronic blockade of OT/AVP receptors during this period decreased the frequency of spontaneous EPSCs in slices of young adults further argue in favor of a role of the peptides in glutamatergic synapse establishment. The decrease in frequency may result from either a change in presynaptic properties or most probably a decrease in the number of active synapses.

In conclusion, our results show that glutamate release, probably by incoming afferents, and dendritic neuropeptide release are both required for the transient increase in the dendritic arbor during development of SON neurons. A developmental role for OT and AVP has been suggested previously (Carter et al., 1993) because several brain areas display a transient increase in OT/ AVP receptors during development (Tribollet et al., 1991a,b) and AVP increases neurite outgrowth in Xenopus (Brinton and Gruener, 1987) and rat hippocampal and cortical cultured neurons (Brinton et al., 1994; Chen et al., 2000; Tarumi et al., 2000).
However, direct evidence for a neurotrophic role of endogenous peptides was lacking. Here we show that, during development, OT/AVP are involved in dendritic plasticity of their secreting neurons and probably play an important role in synaptogenesis, particularly by acting retrogradely on glutamatergic endings.

\section{REFERENCES}

Bodnarenko SR, Chalupa LM (1993) Stratification of ON and OFF ganglion cell dendrites depends on glutamate-mediated afferent activity in the developing retina. Nature 364:144-146.

Brinton RD, Monreal AW, Fernandez JG (1994) Vasopressin-induced neurotrophism in cultured hippocampal neurons via V1 receptor activation. J Neurobiol 25:380-394.

Brinton RE, Gruener R (1987) Vasopressin promotes neurite growth in cultured embryonic neurons. Synapse [Erratum (1988) 2:107] 1:329-334.

Carter DA, Fai CK, Murphy D (1993) Neurohypophyseal peptides as regulators of growth and development. A review. J Mol Neurosci 4:11-19.

Chen Q, Patel R, Sales A, Oji G, Kim J, Monreal AW, Brinton RD (2000) Vasopressin-induced neurotrophism in cultured neurons of the cerebral cortex: dependency on calcium signaling and protein kinase $\mathrm{C}$ activity. Neuroscience 101:19-26.

Chevaleyre V, Dayanithi G, Moos FC, Desarménien MG (2000) Developmental regulation of a local positive autocontrol of supraoptic neurons. J Neurosci 20:5813-5819.

Chevaleyre V, Moos FC, Desarménien MG (2001) Correlation between electrophysiological and morphological characteristics during maturation of rat supraoptic neurones. Eur J Neurosci 13:1136-1146.

Cline HT (2001) Dendritic arbor development and synaptogenesis. Curr Opin Neurobiol 11:118-126.

Dayanithi G, Sabatier N, Widmer H (2000) Intracellular calcium signalling in magnocellular neurones of the rat supraoptic nucleus: understanding the autoregulatory mechanisms. Exp Physiol 85:75S-84S.

Fitzsimonds RM, Poo MM (1998) Retrograde signaling in the development and modification of synapses. Physiol Rev 78:143-170.

Hatton GI (1990) Emerging concepts of structure-function dynamics in adult brain: the hypothalamo-neurohypophysial system. Prog Neurobiol 34:437-504.

Hatton GI (1997) Function-related plasticity in hypothalamus. Annu Rev Neurosci 20:375-397.

Hussy N, Boissin-Agasse L, Richard P, Desarménien MG (1997) NMDA receptor properties in rat supraoptic magnocellular neurons: characterization and postnatal development. Eur J Neurosci 9:1439-1449.

Inglis FM, Furia F, Zuckerman KE, Strittmatter SM, Kalb RG (1998) The role of nitric oxide and NMDA receptors in the development of motor neuron dendrites. J Neurosci 18:10493-10501.

Jontes JD, Buchanan J, Smith SJ (2000) Growth cone and dendrite dynamics in zebrafish embryos: early events in synaptogenesis imaged in vivo. Nat Neurosci 3:231-237.

Kalb RG (1994) Regulation of motor neuron dendrite growth by NMDA receptor activation. Development 120:3063-3071.

Kirov SA, Sorra KE, Harris KM (1999) Slices have more synapses than perfusion-fixed hippocampus from both young and mature rats. J Neurosci 19:2876-2886.

Koester SE, O'Leary DD (1994) Development of projection neurons of the mammalian cerebral cortex. Prog Brain Res 102:207-215.

Kombian SB, Mouginot D, Pittman QJ (1997) Dendritically released peptides act as retrograde modulators of afferent excitation in the supraoptic nucleus in vitro. Neuron 19:903-912.

Kombian SB, Mouginot D, Hirasawa M, Pittman QJ (2000) Vasopressin preferentially depresses excitatory over inhibitory synaptic transmission in the rat supraoptic nucleus in vitro. J Neuroendocrinol 12:361-367.

Lendvai B, Stern EA, Chen B, Svoboda K (2000) Experience-dependent plasticity of dendritic spines in the developing rat barrel cortex in vivo. Nature 404:876-881.

Li Z, Van Aelst L, Cline HT (2000) Rho GTPases regulate distinct aspects of dendritic arbor growth in Xenopus central neurons in vivo. Nat Neurosci 3:217-225.

Nelson EE, Alberts JR, Tian Y, Verbalis JG (1998) Oxytocin is elevated in plasma of 10-day-old rats following gastric distension. Brain Res Dev Brain Res 111:301-303.

Parpura V, Haydon PG (2000) From the cover: physiological astrocytic calcium levels stimulate glutamate release to modulate adjacent neurons. Proc Natl Acad Sci USA 97:8629-8634.

Purpura DP (1975) Dendritic differentiation in human cerebral cortex: normal and aberrant developmental patterns. Adv Neurol 12:91-134.

Rajan I, Cline HT (1998) Glutamate receptor activity is required for normal development of tectal cell dendrites in vivo. J Neurosci 18:7836-7846. 
Stern JE, Armstrong WE (1998) Reorganization of the dendritic trees of oxytocin and vasopressin neurons of the rat supraoptic nucleus during lactation. J Neurosci 18:841-853.

Tarumi T, Sugimoto Y, Chen Z, Zhao Q, Kamei C (2000) Effects of metabolic fragments of $[\operatorname{Arg}(8)]$-vasopressin on nerve growth in cultured hippocampal neurons. Brain Res Bull 51:407-411.

Theodosis DT, Montagnese C, Rodriguez F, Vincent JD, Poulain DA (1986) Oxytocin induces morphological plasticity in the adult hypothalamo-neurohypophysial system. Nature 322:738-740.

Theodosis DT, el Majdoubi M, Gies U, Poulain DA (1995) Physiologically-linked structural plasticity of inhibitory and excitatory synaptic inputs to oxytocin neurons. Adv Exp Med Biol 395:155-171.

Theodosis DT, El Majdoubi M, Pierre K, Poulain DA (1998) Factors governing activity-dependent structural plasticity of the hypothalamoneurohypophysial system. Cell Mol Neurobiol 18:285-298.

Tribollet E, Goumaz M, Raggenbass M, Dreifuss JJ (1991a) Appear- ance and transient expression of vasopressin and oxytocin receptors in the rat brain. J Recept Res 11:333-346.

Tribollet E, Goumaz M, Raggenbass M, Dubois-Dauphin M, Dreifuss JJ (1991b) Early appearance and transient expression of vasopressin receptors in the brain of rat fetus and infant. An autoradiographical and electrophysiological study. Brain Res Dev Brain Res 58:13-24.

Ugrumov MV (1992) Development of the hypothalamic monoaminergic system in ontogenesis. Morpho-functional aspects. Zool Sci Tokyo 9:37-45.

van den Pol AN, Wuarin JP, Dudek FE (1990) Glutamate, the dominant excitatory transmitter in neuroendocrine regulation. Science 250:1276-1278.

Wong WT, Faulkner-Jones BE, Sanes JR, Wong RO (2000) Rapid dendritic remodeling in the developing retina: dependence on neurotransmission and reciprocal regulation by Rac and Rho. J Neurosci 20:50245036. 